\title{
AgNOR measurements as indices of proliferation, ploidy and prognosis
}

\author{
J C E Underwood
}

Like many other developments with diagnostic potential in medical science, interest in AgNORs (silver binding nucleolar organiser regions) is following a predictable cycle: an initial wave of enthusiasm, then scepticism as problems emerge, with only limited applications in prospect.

The publication by Ploton et $a l^{1}$ of a simple staining method enabled relatively easy demonstration of the argyrophilic proteins associated with NORs in interphase nuclei in sections of routinely processed, formalin fixed tissue. This was followed by numerous reports of the AgNOR status of a variety of lesions, notably those in which the distinction between benign and malignant tumours or between different prognostic groups was difficult using ordinary histological criteria. ${ }^{2}$ This resulted eventually in the recognition of our main problems:

(1) There was controversy about what should be counted as an AgNOR. In interphase nuclei the NORs are usually clustered within one or more nucleoli. The individual AgNOR dots may not be discernible because they appear to coalesce, leading to interobserver disagreements and underestimates of the actual numbers.

(2) In many studies, even though there were differences in AgNOR indices (mean numbers of AgNOR dots per nucleus or mean AgNOR area per nucleus) statistically significant at the $5 \%$ level, the numerical ranges between different groups (for example, benign versus malignant) overlapped sufficiently to render the technique a relatively weak discriminant when used prospectively in the routine investigation of genuine borderline cases.

(3) Counting dots in nuclei was so timeconsuming and tedious that few investigators assessed a sufficient number of nuclear profiles to yield a mean AgNOR estimate with the 5\% precision customarily regarded as adequate.

(4) Irrespective of their diagnostic utility, there was doubt about whether differences in AgNOR indices were attributable to cellular proliferation or nuclear ploidy or both.

Because of these problems and uncertainties, advocates of AgNOR methods in pathology are frequently challenged by critical statements such as "NORs are difficult to identify, timeconsuming to count and do not have a consistently proven correlation with other meas- ures of proliferative activity or prognosis"3 to which Trerè responds in the August issue of Clinical Molecular Pathology. ${ }^{4}$

Before dealing with the fundamental question about whether the numbers of interphase AgNOR dots reflect ploidy or proliferation, it must be emphasised that the numbers of discernible (and therefore countable) dots depend on at least five variables:

- the actual number of NOR sites in the nucleus;

- the level of rRNA transcriptional activity;

- the degree NOR dispersion within the nucleus;

- the spatial resolution of the optical system (microscope coupled to either image analyser or human observer);

- control of the AgNOR staining technique.

In the human genome NORs are located on only the five acrocentric chromosomes. The numbers of AgNOR dots could, therefore, reflect ploidy variations but only directly if the variations involved deletion or addition of NOR bearing acrocentric chromosomes. Now, however, numerical chromosome aberrations can be determined in interphase nuclei with more specificity by other methods such as fluorescence in situ hybridisation, although this technique is not yet developed to the extent that it can be applied reliably to sections of tissue processed routinely for diagnostic histopathology. The ability to use routine sections remains a distinct advantage of AgNOR methods.

Transcriptional activity determines the size and therefore microscopic detectability of AgNOR dots; functionally inactive cells have small nucleoli and minute AgNOR dots within them because little rRNA is being generated. Dispersion of NORs occurs with dissolution of the nucleolus immediately before mitotic division. The AgNOR dots, instead of being tightly clustered in one or more nucleoli, separate and scatter through the nucleoplasm, rendering each of them more visible as separate particles; this results in an apparent increase in the numbers of AgNORs. If AgNOR determinations were of widely proven utility in diagnosis or prognostication, considerations of dispersion and transcription might be less relevant in practice.

Recognition of these problems resulted in proposals for the application of standard 
methods for AgNOR quantification, in the hope that this would lead to greater consistency. ${ }^{5}$ In an attempt to overcome the tedium of dot counting and interobserver disagreements about whether a dot is a single AgNOR or a coalescence of two or more, several investigators have used image cytometry to quantify more objectively the AgNOR status of lesions. First, because the pixel resolution of most image analysis systems is too low and, second, AgNOR dots lie at different levels in the nuclear section to enable reliable counting of the numbers individual dots, image cytometry publications report measurements of the area of the AgNOR profile. ${ }^{6}$ Automatic image cytometry of AgNOR areas should be more objective and free of observer bias other than that involved in setting optical density thresholds; dot counts performed by histopathologists, sufficiently experienced to be able to diagnose the lesion from the faintly visible tissue architecture in AgNOR stained sections, may be biased in their interpretation of an AgNOR aggregate as a cluster containing the anticipated number of dots. The only possible caveat in AgNOR area measurements is that they are likely to be influenced by transcriptional activity independent of proliferation. This is because transcriptionally active but mitotically quiescent cells may have higher mean AgNOR areas in their nuclei than those which are "resting".

Regarding whether AgNOR indices reflect proliferative activity or ploidy, the balance of evidence suggests the former, although ploidy variations can be a confounding factor. There is good evidence from in vitro systems and from studies of neoplasms that AgNOR counts and areas correlate more closely with other well accepted measures of proliferation than with ploidy. ${ }^{2}$

Contrary to the impression given by Barnes and Gillett $^{3}$ and by others, there are many examples of AgNOR determinations yielding diagnostically and prognostically useful information. Clear separation exists in the AgNOR ranges of - for example, benign and malignant mesothelial lesions ${ }^{7}$ and melanocytic lesions. ${ }^{8}$ The fact that AgNOR measurements are not more widely adopted, if at all, in routine histopathology reflects the supreme medical utility of the expert (but subjective) diagnostic interpretation of histological or cytological features and the lack of multidisciplinary studies reporting the outcome of clinical trials in which treatment decisions are based at least partly on prognostic groups defined by AgNOR indices. Finally, of course, AgNOR area measurements and laborious dot counting as indices of cell proliferation have been superseded largely by immunostaining for substances such as Ki67 (proliferation "antigens") associated more specifically with the mitotic cycle. Transcriptionally active cells in $G_{0}$ display AgNOR dots but should not normally express proliferation antigens.

1 Ploton D, Menager M, Jeanesson P, Himber G, Pigeon F, Adnet J. Improvement in the staining and visualisation of
the argyrophilic proteins of the nucleolar organiser region at the optical level. Histochem $\mathcal{f} 1986 ; 18: 5-14$.

2 Crocker J. Nucleolar organiser regions. In: Underwood JCE, ed. Pathology of the nucleus. Current Topics in Pathology. Vol ed. Pathology of the nucleus. Current Topics in
82. Berlin: Springer Verlag, 1990:91-149.

3 Barnes DM, Gillett CE. Determination of cell proliferation. f Clin Pathol: Mol Pathol 1995;48:M2-5.

4 Trerè D. Quantitative analysis of silver stained nucleolar organiser regions (AgNORs): a reliable marker of cell proliferation and a promising prognostic parameter in tumour pathology. 7 Clin Pathol: Mol Pathol 1995;48:M219.

5 Crocker J, Boldy DAR, Egan MJ. How should we coun AgNORs? Proposals for a standardised approach. $f$ Pathol 1989;158:185-8.

6 Ruschoff J, Plate KH, Contractor H, Kern S, Zimmermann R, Thomas C. Evaluation of nucleolus organiser regions (NORs) by automatic image analysis: a contribution of (NORs) by automatic image analysis: a con
standardization. $\mathcal{f}$ Pathol 1990;161:113-18.

7 Ayres JG, Crocker J, Skilbeck NQ. Differentiation of malignant from normal and reactive mesothelial cells using the argyrophil technique for nucleolar organiser region associated proteins. Thorax 1988;43:366-70.

8 Crocker J, Skilbeck N. Nucleolar organiser region associated proteins in cutaneous melanocytic lesions: a quantitative study. $f$ Clin Pathol 1987;40:885-9.

9 Wu CC, Woods AL, Levison DA. The application of immunohistochemistry in assessment of cellular proliferation. In: Hall PA, Levison DA, Wright NA, eds. The assessment of cell proliferation in clinical practice. London: Springer of cell proliferation in
Verlag, 1992:141-59. 